\title{
The Evaluation of EV Energy Consumption and Emission and Its Comparison of at Home and Abroad
}

\author{
Siyu Pan ${ }^{1, a}$ \\ ${ }^{1}$ North China Electric Power University, Beijing,102206 \\ aalanncepu@foxmail.com
}

Keywords: Security efficiency; Linear process; Regression analysis; Queue flowing model

\begin{abstract}
This paper analyzed energy consumption and emission estimation method of Blade Electric Vehicles(BEV) both in home and abroad at first, compare its similarity and difference; Then we analyzed consumption and emission of HEV and PHEV, carefully compared the difference between China and America and drawn a conclusion that China is worse than America; At last we gave a brief introduction of EREV's consumption and emission analysis.
\end{abstract}

\section{Introduction}

As the breakthrough of Automotive battery technology, to save energy and reduce emissions, many country's start to research and develop the electric vehicles(EV) these years. In developing country like China, the concern about consumption and emission of EV is special. Thus, it's essential to investigate the standard of EV consumption and emission both in home and abroad, and find out the difference, which can be based on to improve development in China. Establishing a good standard of vehicle energy emission evaluation makes for The development of the market and the innovation of important technology guidance, which has great significance to the development of EV in China.

\section{Blade Electric Vehicle}

Energy Consumption Evaluation Method at Domestic and Overseas. at the present stage, it is same that consumption evaluation method of BEV in home and abroad, respectively from Vehicle consumption power、 thermal efficiency(drive efficiency) and specific energy consumption.

Vehicle consumption power can be calculated from

$$
\mathrm{P}_{\mathrm{d}}=\frac{G f v_{a}}{3.6}+\frac{G i v_{a}}{3.6}+\frac{C_{D} A v_{a}{ }^{3}}{76.14}+\frac{W_{m} v_{a}}{3.6} \frac{\mathrm{d} v}{\mathrm{~d} t}
$$

This Equ. 1 follows the consumption evaluation method of fuel vehicle.

The thermal efficiency is calculated by calculating the average coal-fired power generation. Considering transmission losses house supply、The proportion of power generated by coal-fired power generation、Electric car battery pack efficiency, Motor and transmission system efficiency and other factors, finally get the efficiency of electric vehicle ${ }^{[2]}$.

The specific energy consumption is defined as energy consumption rate divide total weight of vehicle, can be evaluated by The AC electricity consumption of the electric vehicle per kilometer or The average amount of DC power consumed per kilometer.

Emission Evaluation Method at Domestic. At the present stage, it is same that emission evaluation method of BEV in home and abroad, mainly take the Increased gas emissions from coal-fired power plants into consideration.

By calculating the average coal-fired power generation. Considering transmission losses , house supply、 Battery charge and discharge loss 、 energy conversion efficiency and so on, convert electricity consumption per $100 \mathrm{~km}$ into Standard coal quantity of heat-engine plant. According to related documents of the NDRC ${ }^{[3]}$ we can get all kinds of exhaust gases per $100 \mathrm{~km}^{[4]}$.

Emission Evaluation Method at Overseas. From the perspective of the entire life cycle of EV, which includes GreenHouse Gas(GHG) emissions during the driving period and greenhouse gas emissions 
during the manufacturing period.

Because of the independence between the state and the state, the state grid makes the emissions different. Published by the U.S. environmental protection agency,2015C; HIS $2015^{[5]}$.

The emissions in the manufacturing period root in materials and manufacturing processes for lithium batteries. It's shown that by six months of driving BEV we can 'repay' this emission.

From the perspective of the entire life cycle of EV, the emissions are still much lower than fuel vehicle.

\section{Hybrid Electric Vehicle and Plug-in Hybrid Electric Vehicle}

Introduction. Because HEV electric energy comes from regenerative braking, SOC is fluctuating in a small scale. There is a linear relationship between electric consumption and fuel consumption ${ }^{[6]}$. we often convert electric consumption into fuel consumption, then analyses as the traditional fuel car. Below mainly talk about the energy consumption and emission evaluation of PHEV ${ }^{[7]}$.

Energy Consumption Evaluation Method at Domestic. Test Cycle: NEDC cycle at domestic, divided into urban conditions and suburb conditions. Regarded as steady state condition.

Test Mode and Test Routine: As various kinds of modes PHEV owns, we use SAE J1711-1999 of USA, to test every feasible working condition. As for test routine, we now choose:

Two stage test (CD \& CS), carry out condition A and condition B test respectively. When consumption of PHEV is not linear, the condition A test is not accurate. Condition B allows consumption of electric energy, which is not strict enough.

Continued mileage testing, only has an indicator of AER. And it's not so objective and comprehensive.

Fuel consumption tests: use carbon balance method (calculate fuel consumption according to compound emissions). Because in CS there remains electric consumption and China don't amend fuel consumption, the results don't reflect the essence of PHEV working in CS.

Power consumption test, for there is only one NEDC cycle, the result is not accurate.

Result Dispose: It follows the rule of Europe, weighting the result of maximum charging test and minimum charging test. The fuel consumption of CS is showed below:

$$
C=\frac{D_{e} * C_{1}+D_{a v} * C_{2}}{D_{e}+D_{a v}}
$$

But the value of the weight coefficient still exists some problems.

\section{Energy Consumption Evaluation Method at Overseas}

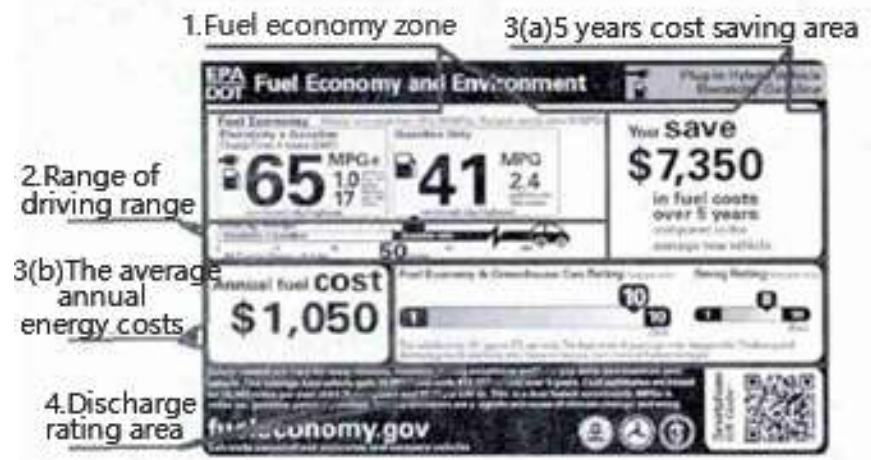

Figure 1. The labels of energy emission evaluation

This label can basically make comments to a PHEV. This is more systematic and advanced than which in China.

1Test Cycle: USA use five working conditions in SAE J1711-1999. Regarded as transient state condition, which is closer to factual loss.

2 Test Mode and Test Routine: For test mode, Europe and Japan both use several minority typical working conditions. Can both simplify test procedure and reflect actual performance. As for test routine, 
USA now choose:

3Two stage test (full electrical test and battery maintenance test). In battery maintenance test there is a regulation that net change in electrical energy can't exceed 1\% from beginning to end.

4Continued mileage testing, including Energy consumption cycle mileage, Actual mileage of electricity consumption and AER.

5Fuel consumption test: use carbon balance method, slope correction method and regression method. It can reflect the essence of PHEV working in CS.

6 Power consumption test, include every cycle of $\mathrm{CD}$. Divide the AC power equally between each test cycle. Results are authentic and credible.

7 Result Dispose

Weight coefficient is based on the use coefficient of travel characteristics of American residents(UF). The fuel consumption of CS is showed below:

$$
F_{\text {fuel }}=U_{\mathrm{f}}\left(R_{\mathrm{CDC}}\right) F_{\mathrm{CD}}+\left[\left(1-U_{\mathrm{f}}\left(R_{\mathrm{CDC}}\right)\right) F \mathrm{Cs}\right]
$$

\section{Erev}

The experiment aiming at the consumption and emission of EREV barely exist. From the existing documents, the test mode is under the above-mentioned test cycle. Imitating research of HEV and PHEV.

\section{Conclusion}

This paper focuses on energy consumption and emission assessment of EV, analyzed and compared the evaluation method of different kinds of vehicles at home and abroad. Draw a conclusion that at present the evaluation of BEV, Chinese is similar to which in foreign countries. but at the evaluation of HEV and PHEV, there still is gap between foreign countries and China.

\section{References:}

[1] ZhenPoWang,Feng ChunSun. Analysis of energy consumption distribution and influencing factors of electric vehicles[J]. Journal of Beijing institute of technology, 2004, (04):306-310.

[2] ShengGuo,QinShi, YanBaoLi,NingGuo. Comparison and analysis of energy consumption and emission of pure electric vehicles and conventional vehicles[J]. Baic Motor Corporation,2014, (01):20-23.

[3] 《Energy Conservation and Environmental Protection》 National Development and Reform Commission

[2004]2505[OL/EB].http://www.moc.gov.cn/2006/jiaotongjj/07jiaotjnw/zhengcefg/guojiazc/200703/t 20070312_197789.htm,2010.7.2.

[4] LeiZhang,TingXu,DaoZhuoJiang, YiQiaoLiang. Comparative analysis of the emission of electric vehicles and traditional vehicles [J]. Zhejiang Electric Power,2012,31(02):57-60.

[5] Tesla baijia. ucsusareport: Why is electric cars more environmentally friendly [DB/OL]? http://auto.gasgoo.com/News/2016/05/2210071371360360005395.shtml.

[6] KongJianQin,HaiFengChen,MaoDongFang,ChunLongZhang. Research on emission and energy consumption evaluation of plug-in hybrid electric vehicle [J]. automotive engineering,2010, (07):11-16.

[7] CongHou,HeWuWang,MingGaoOuYang. Comparative study of PHEV energy consumption evaluation method in China and America [J]. automotive engineering,2015,37(01):1-8.

[8] ChengQiLiu,LaiQingXie, YueZhenFan,LongChen, YuGongLuo. Energy consumption test and analysis of the north winter condition of an electric motor vehicle[J]. automotive engineering,2016, 
(02):45-49.

[9] FanJingBu, YaoNanWang. The fuel consumption rate optimization of electric vehicle improved based on the differential evolution algorithm $[\mathrm{J}]$. Journal of electronic measurement and instrumentation,2016,30(02):200-208.

[10] HanBingWei,DaTongQin,ZhiYuanPeng,ShuJiangChen. HEVThe influence of electric heating energy on the conversion efficiency, fuel consumption and emission of the three-component catalysts [J]. China Mechanical Engineering,2013,24(18):2533-2538. 